\title{
On the periodic variations of secondary cosmic rays and the geomagnetic Pc4 pulsations in BMAr
}

\author{
I. M. Martin ${ }^{1}$, A. A. Gusev ${ }^{1}$, K. Kudela ${ }^{1,2}$, J. Kassovicova ${ }^{2}$, G. I. Pugacheva ${ }^{1}$ \\ ${ }^{1}$ Unicamp-Instituto de Fisica, Depto. de Raios Cosmicos e Cronologia, C.P.6165, 13083-970, Campinas, SP, Brazil \\ ${ }^{2}$ Institute of Experimental Physics, Slovak Academy Sciences, Watsonova 47, 04353 Kosice, Slovakia
}

Received: 4 November 1996 / Revised: 24 February 1997 / Accepted: 5 March 1997.

\begin{abstract}
In a set of balloon flights in the Brazilian magnetic anomaly region (BMAr) short time periodic variations were observed, i.e. pulsation, of secondary charged and neutral particle fluxes, $\mathrm{X}$ - and $\gamma$-ray fluxes with amplitudes of about $2-4 \%$. The pulsations are accompanied by the geomagnetic Pc4 pulsations and have similar periodicity. The phenomenon was observed over various local times and in quiet and disturbed magnetospheric conditions. One of the explanations of this effect, i.e. periodic variation of local cut-off rigidity, and following pulsations of primary and secondary cosmic ray intensity is suggested.
\end{abstract}

\section{Introduction}

Temporal variations of charged particle fluxes and neutral radiation in the Brazilian magnetic anomaly region (BMAr) using balloon measurements have been the subject of systematic studies for more than two decades. In the earliest of these experiments the existence of short periodic variations (pulsations) of secondary charged particle and $\gamma$-ray count rates was revealed in slightly disturbed magnetosphere (Trivedi et al., 1973. Martin et al., 1974). These variations were non-harmonic, but strictly periodic in character. In those works short-periodic fluctuations of geomagnetic field were observed simultaneously with apparent periodicity in the count rates of charged particles and $\gamma$ rays. The time scale of periodicity appeared similar to the scale of geomagnetic Pc4-6 pulsations. These two experiments first showed that a connection exists between geomagnetic pulsations and particle flux periodic variations in the upper atmosphere. The connection was then attributed to the trapped particle precipitation

Correspondence to: G. I. Pugacheva caused by an adiabatic increase and decrease of the altitude of the charged particle mirror points.

In this study we consider several new experimental results concerning short periodic variations of particle fluxes at the balloon flight altitudes in the BMAr, and we attempt to determine whether the particle count rate pulsations are connected with trapped particle precipitation due to geomagnetic field Pc4 pulsations as was supposed earlier. We first consider briefly what is known now about geomagnetic pulsations following Jacobs' (1970) work.

Geomagnetic pulsations with periods less than the characteristic times of geomagnetic storms appear in the magnetosphere as a result of plasma instabilities or as responses to fluctuation in the solar wind. These pulsations, when they pass through the ionosphere, can be registered on the Earth's surface. Some of these oscillations are regular, quasi stable and have quasi sinusoidal form. They are called Pc (pulsation continuous) pulsations. The ranges of pulsation periods are: Pc1-0.2-5 s; Pc2-5-10 s; Pc3-10-45 s; Pc4-45-150 s; Pc5-150-600 s; Pc6->600 s. In the modern understanding $\mathrm{Pc}$ is mostly a manifestation of hydromagnetic wave activity generated by physical processes resulting mainly from interaction of solar wind plasma with the Earth's magnetic field. Studying Pc3, 4 pulsations at low latitudes ( $L=1.4-1.8)$ Ziesolleck et al., (1993) came to the conclusion that the pulsation origin is essentially consistent with the generation of magnetic field line resonances coupled, most likely, with the global compressional modes in the magnetosphere. Pc3 and Pc4 are more abundant type of geomagnetic pulsations, registered on the Earth's surface. They can continue for hours. The Pc3, Pc4 frequently appear simultaneously with a pulsation intensity of the polar aurora, of bremsstrahlung X-rays of auroral electrons, etc. The amplitudes of the Pc4 pulsations increase from equatorial region where they may reach the value of about 1-2 $\gamma\left(1 \gamma=1 \mathrm{nT}=10^{-5}\right.$ Gauss $)$, to the middle latitudes and reach several $\gamma$ at about $55-62^{\circ}$ geomagnetic latitudes and several tens of $\gamma$ at latitudes of $65-68^{\circ}$. 
The longitudinal extent of the pulsations is about $20-30^{\circ}$ at high latitudes and about $120^{\circ}$ at middle latitudes and sometimes these pulsations are global.

\section{Experimental results}

During the numerous balloon experiments performed over South America after the first works of Trivedi et al. (1973) and Martin et al. (1974) periodic variations of charged and even neutral radiation were also observed. In a balloon flight into BMAr on 14 April 1981 Pinto and Gonzales (1989) observed time variations of several tens keV X-ray fluxes with periodicity of about $110 \pm 10$ s, i.e. on the same time scale as Pc4 pulsations. They linked variations of the X-ray fluxes with bremsstrahlung radiation of $\mathrm{keV}$ electrons precipitating into the ambient atmosphere from the inner radiation zone. In their paper, the observed periodicity in X-ray flux was attributed to a more complicated effect, namely to whistler-mode hiss-electron resonance modulated by geomagnetic pulsations occurring at the plasmapause where the hiss is thought to be generated.

During the balloon flight on May 20th 1988 over Campinas $\left(23^{\circ} \mathrm{S}, 47^{\circ} \mathrm{W}\right)$, Brazil, short period variations of $>5 \mathrm{MeV}$ electron flux and of $>30 \mathrm{MeV}$ proton flux at equivalent altitudes of $23-173 \mathrm{~g} / \mathrm{cm}^{2}$ were detected (Bui Van et al., 1989). A period of $150 \mathrm{~s}(6.7 \mathrm{mHz}$ frequency) with a confidence level of $99.6 \%$ was identified through Fourier analysis of the flux values. The variations were detected during local noon for quiet magnetospheric conditions. The experiment showed that the charged particle pulsations can be observed during quiet magnetospheric conditions and in the deeper layers of atmosphere $\left(173 \mathrm{~g} / \mathrm{cm}^{2}\right)$, where the appearance of precipitating particles is not expected, because of the considerable particle energy $\sim 700 \mathrm{MeV}$ needed to reach this depth. Trapped particles with this enormous energy do exist in the inner zone, but their fluxes are extremely low.

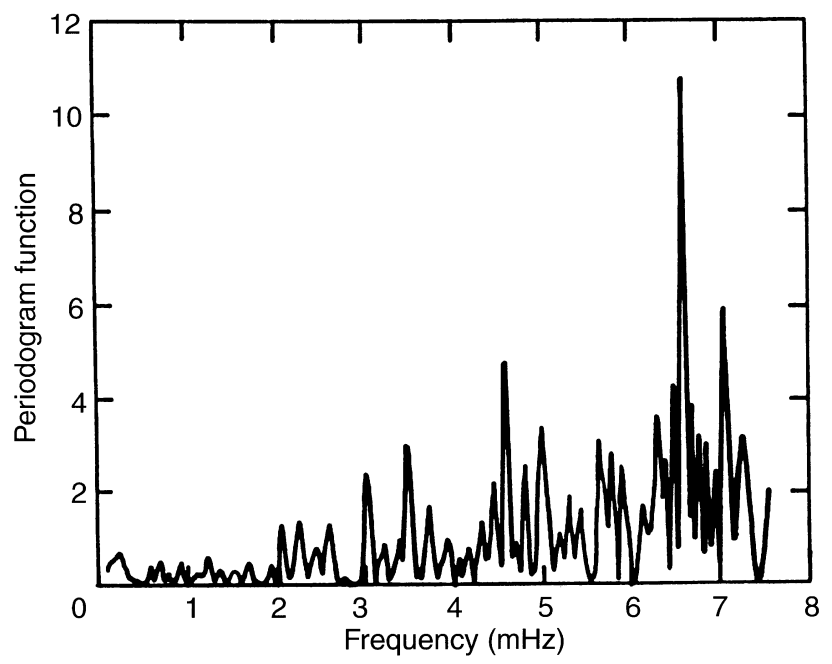

Fig. 1. The power spectrum density of secondary particle fluxes registered on balloon flight over Bauru on 22 April 1989
The new experimental results about relationship between geomagnetic pulsations and particle flux variations at balloon altitudes in the BMAr come from our recent balloon flights over Bauru, Brazil, reported by Bui Van et al. (1993), Bazilevskaya et al. (1994) and over São José dos Campos, Brazil, by Jayanthi et al. (1996).

In the balloon flight over Bauru $\left(22^{\circ} \mathrm{S}, 49^{\circ} \mathrm{W}\right)$ in the evening of 22 April 1989 periodic variations of the charge particle flux were observed (Bui Van et al., 1993). For quiet time magnetospheric conditions charged particle (electrons with energy $\mathrm{E}_{e}>5 \mathrm{MeV}$ and protons with energy $\mathrm{E}_{p}>30 \mathrm{MeV}$ ) flux pulsations were detected with a period of $150 \mathrm{~s}$ at altitudes from 226 up to $10 \mathrm{~g} / \mathrm{cm}^{2}$. The time resolution of the registered fluxes was $20 \mathrm{~s}$ and within this record there was a distinctive modulation frequency of $6.7 \mathrm{mHz}$ (i.e. a period of $150 \mathrm{~s}$ ) at a significance level of $99.6 \%$. In Fig. 1 the power spectrum density (PSD) of the flux variations is shown. In an independent measurements of local ozone concentration performed on the same flight no period similar to $150 \mathrm{~s}$ ( $2.5 \mathrm{~min}$ ) was observed, but a period of $4.5 \mathrm{~min}$ was found. This testifies that the periods observed were not caused by balloon rotations or any other purely technical effects.

The second flight over Bauru was made on 8-9 November 1991 (Bazilevskaya et al., 1994). To register the omnidirectional charged particle flux 100 Geiger counters with an effective area of $18 \mathrm{~cm}^{2}$ were used. For vertical incidence flux measurements they were jointed in 50 telescopes with geometrical factors of $18 \mathrm{~cm}^{2} \mathrm{sr}$. Gamma-ray flux in the energy range $20-2000 \mathrm{keV}$ was registered in five energy channels by four scintillation detectors using $\mathrm{NaI}(\mathrm{Tl})$-crystal technique. For neutron detection 30 neutron counters filled with ${ }^{3} \mathrm{He}$ and covered with polyethylene moderators were used. The $20 \mathrm{~s}$ telemetry time resolution was used for count rate registration permitted revelation of flux pulsations with characteristic times of $\mathrm{Pc} 4$ pulsations.

In Fig. 2 an example of the periodic, but nonharmonic, variations of the registered omnidirectional charged particle flux $\left(\mathrm{E}_{e}>200 \mathrm{keV}\right.$ and $\left.\mathrm{E}_{p}>5 \mathrm{MeV}\right)$ is shown. It shows that these flux variations are far from merely being statistical variations. Fourier-analysis (as proposed by Horne and Baliunas, 1986) of the omnidirectional charged particle flux reveals the existence of

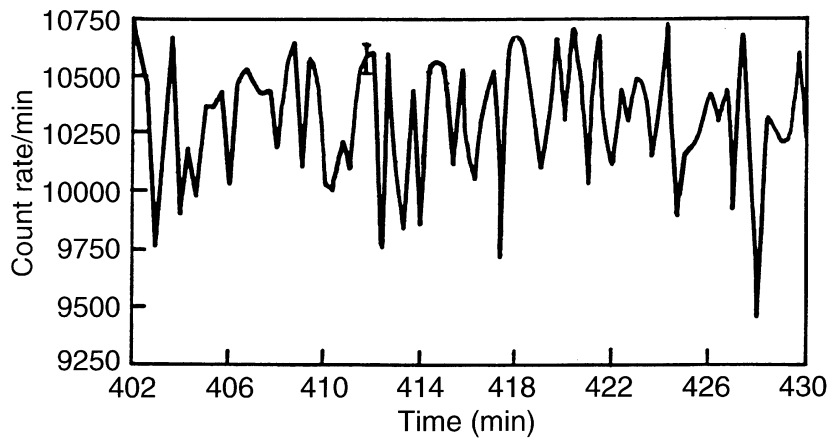

Fig. 2. The time dependence of the omnidirectional charged particle flux registered on balloon flight over Bauru on 8-9 November 1991 


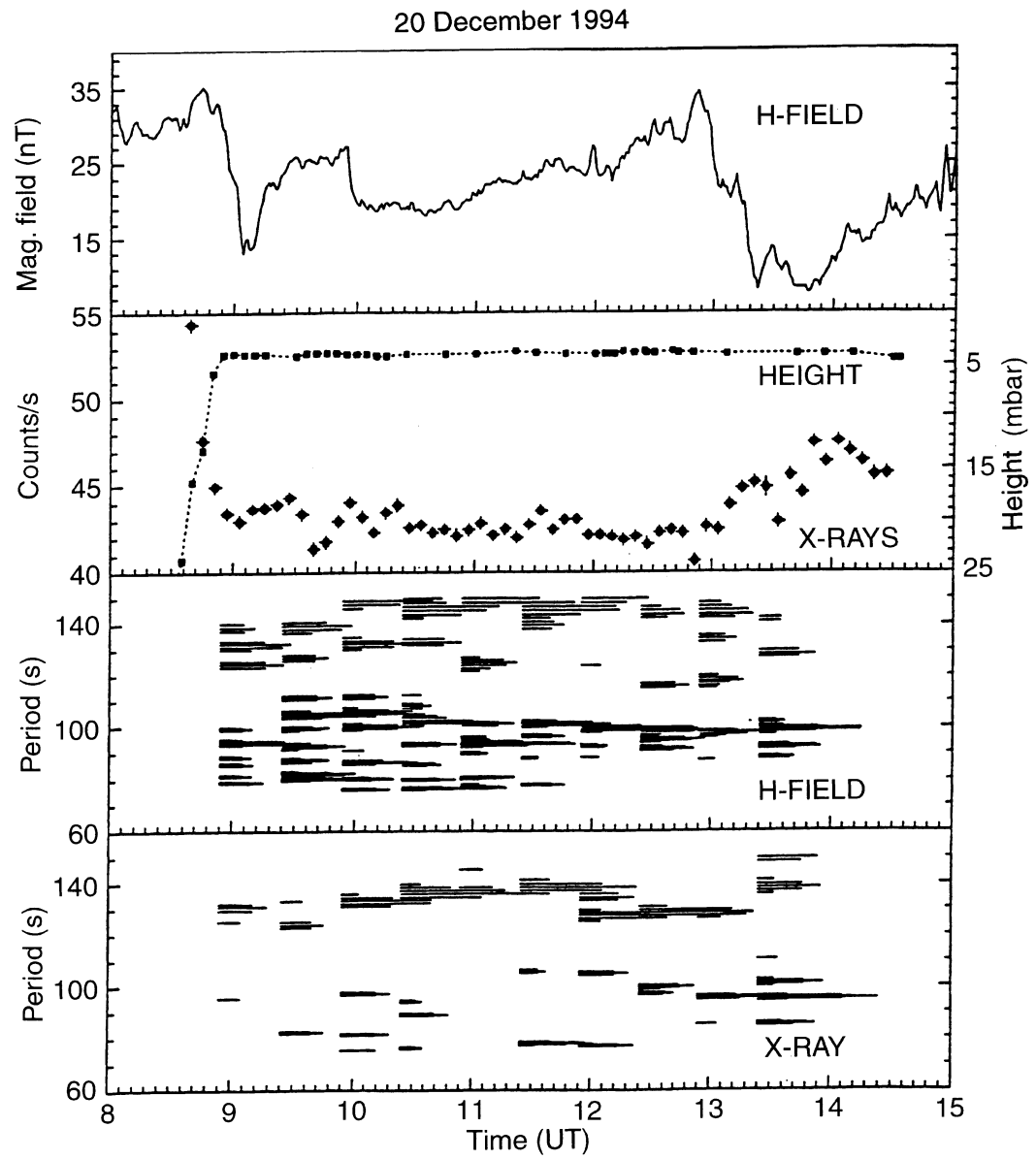

Fig. 3. From top to bottom: the time dependence of $H$-component of geomagnetic field (São José dos Campos); the X-ray count rates; and the periods of Geomagnetic and X-ray flux pulsations observed every half hour during balloon flight of 20 December 1994 periodic pulsations of the energetic charged particle flux with distinctive periods of 1 and $2 \mathrm{~min}$ (Pc4) in the PSD at a significance level higher than $95 \%$. The same analysis also demonstrates the existence of neutron flux pulsations with periods of about $\sim 66 \mathrm{~s}$ and with similar statistical significance. The latter indicates that the particle flux pulsations most likely have nothing to do with the variations of the particle mirror point altitude due to geomagnetic pulsations, because neutrons obviously are not affected by the magnetic field. In this experiment the $0.5-2.2 \mathrm{MeV} \gamma$ ray flux pulsations with a distinct period of $85 \pm 5 \mathrm{~s}$ were also observed. The amplitudes of charged particle, neutron and $\gamma$-ray flux pulsations are of similar magnitude, namely $2-4 \%$. Pulsations of X-rays (with $\mathrm{E}_{x}=20 \mathrm{keV}-140 \mathrm{keV}$ ) and vertical charged particle fluxes (with $\mathrm{E}_{e}>5 \mathrm{MeV}$ and $\mathrm{E}_{p}>30 \mathrm{MeV}$ ) were not so clear. All pulsations were observed from midnight to midday of 9 November 1991 during recovery phase of the moderate geomagnetic storm with $D_{s t}=-98 \mathrm{nT}$.

In the last balloon flight on 20 December 1994 over São José dos Campos, $\left(23.23^{\circ} \mathrm{S}, 45.85^{\circ} \mathrm{W}\right)$ further confirmation of the simultaneous existence of geomagnetic Pc4 pulsations and X-ray $(10-140 \mathrm{keV})$ pulsations with periodicities of about $80-110 \mathrm{~s}$ and $125-150 \mathrm{~s}$ was obtained (Jayanthi et al., 1996). The observations were made for quiet magnetospheric conditions during the morning and midday local time sectors. The count rates of X-rays and geomagnetic field data were registered with high time resolution of $1 \mathrm{~s}$ and $3 \mathrm{~s}$, respectively. This good time resolution permitted an unambiguous analysis of the similar pulsation periods of the both components. Fig. 3 shows the time dependence of the $H$-component of the geomagnetic field, the counting rates of X-rays and the results of periodogram analysis for every half hour interval of both components.

It is well known, that some difficulties exist in the interpretation of results of Fourier analysis and this calls for caution in our analysis. As a rule, this is connected with the sporadic character, and short time duration of pulsations (several periods only), with changes of a pulsation phase, etc. It must be stressed that we observed particle count rate pulsations with similar time variations to the Pc4 periodicity in the various balloon experiments over a total time of about $100 \mathrm{~h}$. Thus, we concluded that at least in equatorial BMA region, at atmospheric altitudes of about $25-40 \mathrm{~km}$, sporadically periodic pulsations of secondary charged and neutral particle, $\gamma$ and X-ray fluxes do appear.

\section{Analysis and discussion}

From all these experiments one important conclusion can be made. The pulsations appear in charged and neutral components, during various local times, independently of the geomagnetic activity, at low (223 and $\left.173 \mathrm{~g} / \mathrm{cm}^{2}\right)$ and at high $\left(3-4 \mathrm{~g} / \mathrm{cm}^{2}\right)$ equivalent atmo- 
spheric depths. The pulsations observed are accompanied by geomagnetic Pc4 pulsations of the same periodicity. From the observations it could be concluded that in the quieter magnetosphere the probability of observing flux pulsations of a larger period of about $2.5 \mathrm{~min}$ is greater and during more disturbed magnetosphere the probability of flux pulsations with a $\sim 1 \mathrm{~min}$ period is greater. The number of balloon experiments carried out to date is still not enough to assert this reliably. But the data from observations already made is suggestive of this relationship.

To discover the mechanism of the flux pulsations we must consider the geomagnetic conditions in the points of measurements. According to the IGRF geomagnetic field model Campinas is located at $L=1.16$ i.e. a closed $L$-shell where according to AP-8, AE-8 proton and electron NASA radiation belt models (Vette et al., 1978) significant trapped particle fluxes of the inner belt still exist at the equatorial top of the geomagnetic field line. Inclination of the magnetic field line to zenith is $68^{\circ}$ and a vertically oriented device registers particles with the pitch-angles are roughly equal to $68^{\circ}$. Mirror points of these particles are located below the Earth's surface, their lifetime is of the order of the atmospheric transit time, and their flux value is essentially zero. So at balloon flight altitudes vertically oriented telescopes can only register a sporadically appearing flux of precipitating particles and an omnidirectional detector registers a trapped flux close to its mirror point. Because of the absorption coefficient of several tens of $\mathrm{keV} \mathrm{X}$-rays and low energy $\gamma$-rays $\lambda \sim 4-10 \mathrm{~g} / \mathrm{cm}^{2}$ in the air (Yuan and $\mathrm{Wu}, 1961)$ bremsstrahlung X-rays can also reach the balloon altitudes from the atmospheric boundary and provide information about trapped and precipitating particles incident to the atmospheric boundary from the equatorial top of the geomagnetic field line of this region.

Let us consider whether the mechanism of the changing of trapped particle mirror point altitude due to adiabatic change in the geomagnetic field strength can explain simultaneous observation of Pc4-6 pulsations and periodic particle flux variations, as was considered practically in all works until the present time.

The characteristic times of trapped particle motion in the geomagnetic field are markedly shorter than the Pc4 periods: the period of Larmour rotation is $2.2 \cdot 10^{-6} \mathrm{~s}$ for $200 \mathrm{keV}$ electrons and $0.003 \mathrm{~s}$ for $5 \mathrm{MeV}$ proton, and the bounce period of particle motion between the mirror points is $0.1-0.2 \mathrm{~s}$ for $\mathrm{MeV}$ electrons and for tens of $\mathrm{MeV}$ protons at $L=1.16$ (Lyons and Williams, 1987). Thus, the electrons and protons move in the geomagnetic field modulated by Pc pulsations with the conservation of the first and second adiabatic invariants. If the geomagnetic field strength decreases on $\Delta B$, then the conservation of the first adiabatic invariant prescribes a decrease of the particle pitch-angle $\alpha_{o}$ as $\Delta B / B=2 \Delta \alpha_{o} / \operatorname{tg} \alpha_{o}$, where $\alpha_{o}$ is a particle pitch-angle at the top of the magnetic line. According to this, the altitude of the mirror point decreases. The amplitude of Pc4 pulsation observed at Campinas is about $2 \mathrm{nT}$, the ambient magnetic field induction is 0.232 Gauss, $\alpha_{o}=68^{\circ}$, then $\Delta B / B \sim 10^{-4}$ and $\Delta \alpha_{o} \sim 0.01^{\circ}$. Thus, at the top of geomagnetic field line a particle pitch-angle oscillates with an amplitude of about $\Delta \alpha \sim 0.01^{\circ}$. Then, at the balloon altitudes the value of the trapped particle flux will be variable too. To provide the observed amplitude of particle count rate variation of $\sim 2-4 \%$, a pitch-angle distribution of trapped particle flux must be extremely narrow, i.e. about $F(\alpha) \sim(\sin \alpha)^{6000}$. But the measurements made by OHZORA (Nagata et al., 1988) show the power of $\sin \alpha$ in the empirical relation $\sin ^{N} \alpha$ is no more than $N=20$ in this region and it could only provide a flux variation amplitude of $\sim 0.01 \%$.

On the other hand, the experiments over Bauru were made at the unclosed geomagnetic $L$-shell 1.13. Due to the low altitude of the latter, trapped particles cannot perform the entire longitudinal drift motion and die in the atmosphere. The AP-8, AE-8 models do not give any appreciable trapped particle fluxes there and we cannot attribute the results to any particle precipitation mechanism including hiss-electron resonance. The observation over Bauru of pulsations of neutron flux, obviously not affected by magnetic field, leads us to the conclusion that the pulsations observed there are likely connected to the secondaries of atmospheric origin produced in the nuclear interactions of cosmic rays (CR) with the residual atmosphere. Following that it is natural to suppose that what is modulated is the source of the secondaries, i.e. the cosmic ray intensity directly.

As a result of this reasoning we arrived at the more likely hypothesis, that in all these mentioned experiments where both charged and neutral particles were registered they were likely to be the secondary particles of cosmic ray origin and therefore their flux pulsations are connected not with local geomagnetic conditions within the inner radiation zone, but rather with primary cosmic ray behavior in the geomagnetic field.

Let us consider this hypothesis in detail.

The relativistic energy CR passes quickly, on a time scale less than $0.1 \mathrm{~s}$, through the part of magnetosphere (from $\sim L=5.0$ to $L=1.0$ ), where geomagnetic field Pc4-6 pulsations sporadically exist (Ziesolleck et al., 1993). Because the pulsation period is of the order of 1000 times greater than time of CR transit motion in magnetosphere, the cosmic particle trajectory depends on pulsation phase. Hence a cut-off rigidity $R_{\mathrm{c}}$ of the observation point also changes with the pulsation phase modulating $\mathrm{CR}$ intensity at this point.

To provide the experimentally observed amplitude of flux pulsation of $2-4 \%$ a cut-off rigidity variation of about $1-2 \%$ is needed. It can be estimated from a relationship (Kudela et al., 1996):

$\Delta I / I=-1.7 \cdot\left(\Delta R_{\mathrm{c}} / R_{\mathrm{c}}\right)$

where $I=I\left(R_{\mathrm{c}}\right) \sim R_{\mathrm{c}}^{-1.7}$ is $\mathrm{CR}$ intensity at an observation point.

To determine the cut-off rigidity in Bauru we numerically computed energetic proton trajectories by solving Lorentz equation with a geomagnetic field that is a sum of internal and external sources (Peredo and Tsyganenko, 1993) and the magnetic field of the Pc4 pulsations. As a pulsation model we adopted the 
perturbation $\Delta B=B_{\text {horiz }}$, model describing horizontal $B$ component in equatorial region: according to Jacobs, (1970) at $L=1.13 B_{\text {horiz }}=2 \mathrm{nT}$; at $L=5 B_{\text {horiz }}=20-60$ $\mathrm{nT}$; at $L \geq 7 B_{\text {horiz }}=0 \mathrm{nT}$.

A precise geomagnetic pulsation model still does not exist, and in this work we would like to highlight the question about the necessity to describe and model the global behavior of Pc4-6 pulsations in the whole magnetosphere.

For numerical modeling we have used a CR trajectory tracing code (Kassovicova and Kudela, 1995). Backward tracing of the proton trajectory from the place of observation with the opposite initial velocity vector and charge was used, in a similar way to the other codes (e.g. Flueckiger et al., 1985; Danilova and Tyasto, 1990). We assumed that the magnetosphere "boundary" is located at 25 Earth's radii, and the trajectory is considered to be allowed if the particle crosses this border to interplanetary space. In the opposite case, the trajectory is considered to be a forbidden one. Finally, the numerical integration of the primary CR spectra over the region $R>9.95 \mathrm{GV}$ with the weight function $\mathrm{p}(R)(\mathrm{p}=1$ for the allowed trajectory, otherwise $\mathrm{p}=0)$ was made. Only vertically incident protons have been taken into account. The step in $\mathrm{d} R$ was taken to be 0.01 $\mathrm{GV}$, and the CR intensity with and without applying $\Delta B$ was obtained.

The amplitude of the variation of primary CR intensity for these two cases is about $0.4 \%$ for $\Delta B=20 \mathrm{nT}$ when the initial altitude of simulated trajectory is at a sea level. With an increase of the initial altitude to $50 \mathrm{~km}$, the magnitude of the CR intensity variation also increases to $0.7 \%$. Increasing $\Delta B=B_{\text {horiz }}$ to $60 \mathrm{nT}$ we obtain the observed order of magnitude of the intensity variation $2-4 \%$. Thus, using the simplified model of the geomagnetic pulsations, we show the importance of the effect of magnetospheric "transparency" to the primary cosmic rays in the observed variability of cosmic ray secondaries at balloon altitudes.

It is natural to expect that this kind of variation must also manifest itself in the neutron monitor (NM) count rates. For many years, temporal variations in $\mathrm{CR}$ intensity have been studied on time scales from 11 years to a few minutes (Kudela and Venkatesan 1993; Kudela, et al., 1996; and others). Analysis of time series of 5-min data of CR intensity from neutron monitors are commonly used. But for this work we need NM data, recorded with higher time resolution of the order of seconds. Fortunately, we found such data at Lomnicky Stit (LS) $\left(49^{\circ} 20^{\prime} \mathrm{N}, 20^{\circ} 22^{\prime} \mathrm{E}, R_{\mathrm{c}}=4.0 \mathrm{GV}\right.$, NM data count rate $-1.510^{5}$ per $5 \mathrm{~min}$ ), recorded from the year 1994 with a time resolution of about $10 \mathrm{~s}$.

Power density analysis of this data has been used for the event period 20 December 1994, when pulsations were observed during a balloon flight over São José Dos Campos (Jayanthi et al., 1996) (see Fig. 3). For the other events mentioned the temporal resolution of the data set was not sufficient. When the whole period (December 20, 1994, 08-15 UT) was taken into account and the analysis was done on an hourly basis, several significant periods of between 50-200 s were seen in the periodograms. However, in most cases, they were of transient, temporary character. To obtain the characteristic pattern of the periodogram, we made a periodogram analysis for the whole event time interval. The result is seen in Fig. 4. The PSD is still complicated, however the strongest peak at $150 \mathrm{~s}$ is persistently observed (the same as observed in all balloon experiments for different epochs) and the two band structure is apparent too: the first interval where $T<110 \mathrm{~s}$ and the second one where $150<T<260$ s. Several peaks in both regions are detected. This two band structure corresponds to the two band structure (80-110 s and 125-150 s) observed also in the profile of secondary X-rays in Fig. 3. On the whole a qualitative agreement is seen in PSD of primary CR sensed by Lomnicky Stit NM, and the X-ray secondaries in the balloon data.

We consider the two band structure of periodograms of the two time series in Figs. 3 and 4 and strongest peak at $150 \mathrm{~s}$ periodicity to be important features, supporting our hypothesis of the changing "transparency" of the magnetosphere to the primaries caused by the Pc4 geomagnetic pulsations.

One reason why a precise quantitative correspondence between the two PSDs is not expected, is the difference of location between the Lomnicky Stit NM $(L=2.0)$ and the São José dos Campos balloon launch site $(L=1.16)$. The asymptotic directions of the Lomnicky Stit neutron monitor are also different from that of São José dos Campos. If the perturbation $\Delta B$ is variable and depending on the geomagnetic position of the observation, the exact correspondence of primary CR PSD at Lomnicky Stit and that of secondary fluxes over São José, is difficult to expect.

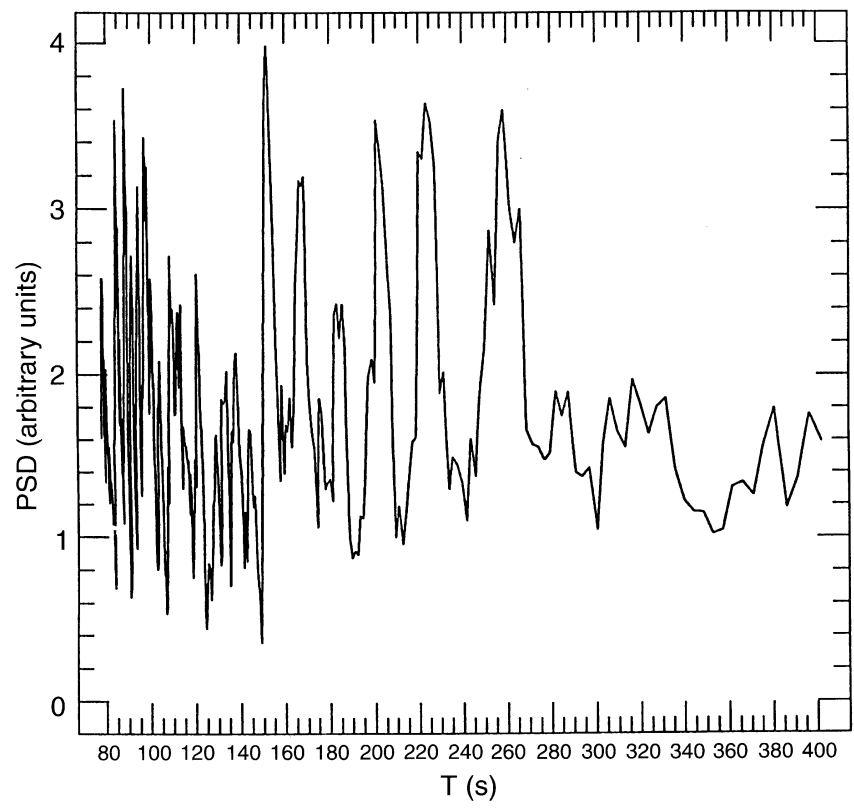

Fig. 4. The power spectrum density of Lomnicky Stit neutron monitor count rate registered on 20 December 1994 


\section{Summary and conclusions}

During the set of balloon flights in the BMAr there were observed charged and neutral secondary particle periodic variations (pulsations) and also X-ray and $\gamma$-ray pulsations with the periods similar to characteristic periods of geomagnetic Pc4 pulsations. The particle and $\mathrm{X}$ - and $\gamma$-ray pulsations are accompanied by geomagnetic Pc4 pulsations. The amplitudes of secondary particle pulsations are about $2-4 \%$. They have been observed during all local times and in quiet and disturbed magnetospheric conditions and also deep into the atmosphere.

We presented here the results of the first analysis of short time scale variations of secondary CR measured in balloon experiments. The analysis has shown that there exist special type of flux variations, i.e. pulsations, that appear intimately connected with geomagnetic Pc4-6 pulsations.

The effect can be explained by periodic variation of the local cut-off rigidity and the consequent modulation of the primary and secondary CR intensities.

It is desirable to continue studying the effect and its relation to geomagnetic pulsations in more detail: for example, the dependence of amplitude and periods of secondary particle flux pulsations and of NM counting rate pulsations on geomagnetic conditions and geomagnetic position of the observation point, i.e. not only in BMA region.

In particular, the verification or rejection of the validity of our suggestion, requires: (1) measurements of secondary particles on balloons in positions close to the neutron monitor observations, and/or (2) checking the "coherence" of the PSD of several neutron monitors with good temporal resolution at different positions. The latter is fundamental to discriminate between the "global" pulsations and effects of anisotropy, which can be seen only in comparison with the PSD of neutron monitors that have substantially different acceptance directions. To understand the influence of local, atmospheric effects on the structure of PSD at high pulsation frequencies (periods less $\sim 80 \mathrm{~s}$ ), comparison of spectra at two neutron monitors with similar acceptance angle structures, e.g. Jungfraujoch and Lomnicky Stit, is expedient. It is interesting also to investigate whether the periodicity of $150 \mathrm{~s}$ permanently exists in NM counting rates and secondary fluxes and what the causes of its time evolution are.

Analysis of this nature can be useful in a systematization of secondary CR fluctuations over different time scales and can be used for detailed study of general characteristics of $\mathrm{CR}$ fluctuations below the diurnal periodicity. In a similar way the general characteristics of the CR time series which reflect interplanetary magnetic field space structure, secondary CR pulsations, probably, reflect aspects of the global magnetospheric structure and its dynamics. Certainly, information about these relationships is needed in further investigation.
Acknowledgements. This work was supported by FAPESP-SP, 93 4978-0 CNPq 522111/96-3, FAEP of Unicamp.

Topical Editor K.-H. Glaßmeier thanks W. N. Spjeldvik for his help in evaluating this paper.

\section{References}

Bazilevskaya, G. A., N. A. Bui-Van, A. N. Kvashnin, I. M. Martin, S. V. Mizin, A. I. Podgorny, Yu. I. Stozhkov, A. Turtelli Jr., Time fluctuations of charge and neutral cosmic-ray component fluxes as measured in the region of the Brazilian Magnetic Anomaly region, Il Nuovo Cimento 17 C (3), 223-235, 1994.

Bui Van, N. A., I. M. Martin, A. Turtelli Jr., Yu. I. Stozhkov, V. V.Sibikin, I. M. Fradkin, and A. Svirzhevskaya, Short-period variation of cosmic ray intensity observed in the stratosphere, $I l$ Nuovo Cimento 12, (6), 811-818, 1989.

Bui Van, N. A., C. A. Vieira, L. P. Borovkov, V. A. Zhavkov, and V. A. Tumanov, Short-period variation of cosmic-ray intensity and ozone density observed in the stratosphere, Il Nuovo Cimento, 16 C, 9-15, 1993.

Danilova, O. A., and M. I. Tyasto Variations of cosmic ray cut-off rigidities, Proc. 21 Int. Cosm. Ray Conf., Adelaide, 7, 6-9, 1990.

Flueckiger, E. O., D. F. Smart, M. A.Shea, L. C.Genhole, and A. A.Bathurst, Estimating the change in asymptotic direction due to secular changes in the geomagnetic field, 19th Int. Cosm. Ray Conf., 5, 336-339, SH 6.1-13, 1985.

Horne J. H. and S. L. Baliunas, A prescription for period analysis of uneverly sampled time series, Astrophys. J., 302, 757-763, 1986.

Jacobs, J. A., Geomagnetic micropulsations, in Physics and Chemistry in Space, Springer, Berlin Heidelberg New York, 1, 179 pp, 1970.

Jayanthi, U. B., M. G. Pereira, I. M. Martin, N. B. Trivedi, and L. L.Lazutin, Substorm associated Pc4 pulsations in X-ray precipitation data of SAA region, 31st Scientific Assembly of COSPAR 1996, Abstr., The University of Birmingham, England, D0.5 session, p. 221, 1996.

Kassovicova J., and K. Kudela, Calculations of trajectory of cosmic rays in the geomagnetic field, Preprint of Tecl. University of Kosice, Slovakia, 1995.

Kudela K., and D. Venkatesan, On scaling temporal variations of cosmic ray intensity, Geophys. Res. Lett, 20, 2793-2796, 1993.

Kudela K., D. Venkatesan, and R. Langer, Variability of cosmic ray power spectra, J. Geophys. Res, accepted, 1996.

Lyons, L. R., and D. J.Williams, Quantitative aspects of magnetospheric physics, D.Reidel, Dordrecht, 1987.

Martin, I. M., D. B. Rai, R. A. R. Palmeira, N. B. Trivedi, M. A. Abdu, and J. M. da Costa, Enhanced low-energy $\gamma$ rays at balloon altitude in the Brazilian magnetic anomaly, Nature, 252, (5478), 25-27, 1974.

Nagata, K., T. Kohno, H. Murakami, A. Nakamoto, N. Hasebe, T. Takenaka, J. Kikuchi, and T. Doke, OHZORA high energy particle observations, J. Geomagn. Geoelectr., 37, 329-345, 1985.

Peredo, M., and N. A. Tsyganenko, GEOPACK 93, package of FORTRAN programs, NASA, 1993.

Pinto, O. Jr., and W. D. Gonzalez, Energetic electron precipitation at the South Atlantic Magnetic Anomaly: a review, J. Atmos. Terr. Phys., 51, (5), 351-365, 1989.

Trivedi, N. B., D. B. Rai, I. M. Martin, and J. M. da Costa, Particle precipitation in the Brazilian Geomagnetic Anomaly during magnetic storms. Planet. Space Scie., 21, 1699-1704, 1973.

Vette, J. I., K. W. Chan and M. J. Teague, Problems in Modeling the Earth's Trapped Radiation Environment NASA-TM-80447, AFGL-TR-78-0130, Washington, D.C., 1978.

Yuan, L. C. L., and C. S. Wu, Fundamental Principles and Methods of Particle Detection, Academic Press, New York, 1961.

Ziesolleck, C. W. S., B. J. Fraser, F. W. Menk, and P. W. McNabb, Spatial characteristics of low-latitude Pc 3-4 geomagnetic pulsations, J. Geophys. Res., 98, (1), A12, 197-208, 1993. 